\title{
Interactive comment on "Evaluating the Lower Tropospheric COSMIC GPS Radio Occultation Sounding Quality over the Arctic” by Xiao Yu et al.
}

\section{Anonymous Referee \#3}

Received and published: 31 October 2017

\section{General comments}

The paper "Evaluating the Lower Tropospheric COSMIC GPS Radio Occultation Sounding Quality over the Arctic" by Yu, Xie, and Ao investigates the usefulness of Radio Occultation ( $\mathrm{RO}$ ) observations for studies of the Arctic lower troposphere. The penetration depth of COSMIC RO sounding above 65 degrees north for different seasons is described, and the uncertainties of the lower-tropospheric RO observations are quantified using two type of uncertainty definitions: the structural uncertainty as quantified from the differences between two processing centers, and the parametric uncertainty as quantified by differences between $\mathrm{RO}$ and alternative data sources, here radio sondes and reanalysis data.

Overall, the results presented are mostly those expected. The impact of humidity 
(mainly during Arctic summer) is line with the general understanding of RO behaviour. However, the study makes an important point in stressing the usefulness of RO for AMTD the lower troposphere in Arctic, where other data sources are scarce. RO data are most likely under-exploited in this type of application, and the manuscript gives a good description of the advantages and disadvantages of using RO data in the Arctic lower troposphere. The potential shortcomings of the RO method for this particular application are pointed out and at least partly explained.

The paper is suited for publication in AMT.

\section{Specific comment}

A question and a suggestion for the future: the parametric uncertainties of the JPL and UCAR data are obtained by identifying RO profiles closer than 3 hours and 300 $\mathrm{km}$ to a radiosonde profile. The statistics (mean, medians, and standard deviations) of the RO-radiosonde differences are plotted. These statistics are influenced both by actual RO-radiosonde differences and by the fact that the two type of observations are not perfectly collocated. We are mainly interested in the first component, while the second component acts to disguise the "true" differences. One could consider using the statistics of the double differences (RO-REANRO)-(RDS-REANRDS), where REANRO and REANRDS are reanalysis data collocated to the RO profiles and the radiosondes, respectively. The impact of an imperfect collocation between $\mathrm{RO}$ and radiosondes is much smaller with this type of comparison using a reanalysis model as a transfer medium. I would like to see a comment from the authors on this subject.

\section{Minor comments}

Abstract, line 10: "predication" should be "prediction". 
Abstract, line 11: in "... demonstrated as a high-quality observation with ..." something appears to be missing. It should be "observation type" or "observation technique" AMTD rather than just "observation".

Abstract, line 12: "high-vertical-resolution" should be "high vertical resolution". It is correctly used in the Introduction, line 4, but not here. I'm not an expert in grammar, but this type of construction is only used as an adjective. As an example, this would be a correct sentence: "a measurement with a high vertical resolution is a high-vertical-resolution measurement."

Abstract, line 18: "Over 70

Introduction, line 1: "twice as much as global average rate" should be "twice as much as the global average rate".

"MetOp" should be "Metop".

Interactive comment on Atmos. Meas. Tech. Discuss., doi:10.5194/amt-2017-230, 2017. 